\title{
Spanish Society of Medical Oncology (SEOM) Cancer and Thrombosis Working Group
}

\author{
Andrés J. Muñoz Martín • Vanesa Pachón Olmos • Miguel Martín Jiménez • Emilio Alba Conejo · Juan Jesús \\ Cruz Hernández, on behalf of SEOM Cancer and Thrombosis Working Group
}

Received: 1 December 2011 / Accepted: 8 December 2011

Currently, venous and arterial thromboembolic disease is considered one of the main causes of morbidity and mortality among cancer patients. Multiple studies have evidenced the association between thromboembolic disease and a poor overall prognosis. Recently, an increased incidence of thromboembolic events of multifactorial aetiology has been observed. Some of the main related causes are improved diagnosis methods and the introduction of new antitumour drugs. Given all of these circumstances, thromboembolic disease is a challenge for all specialists involved in caring for cancer patients and especially for medical oncology specialists. Since 2006, the main clinical oncology societies in the world have paid special attention to this disease, and among their activities we should highlight the various

\footnotetext{
A.J. Muñoz Martín (可) · M. Martín Jiménez

Medical Oncology Service

General University Hospital Gregorio Marañón

C/ Doctor Esquerdo, 46

ES-28007 Madrid, Spain

e-mail: andresmunmar@hotmail.com

V. Pachón Olmos

University Hospital Ramón y Cajal

Madrid, Spain

E. Alba Conejo

Clinical University Hospital Virgen de la Victoria

Málaga, Spain

J.J. Cruz Hernández

Clinical University Hospital

Salamanca, Spain
}

clinical guidelines specific for cancer patients. In 2009, the Spanish Society of Medical Oncology (SEOM) set up a series of initiatives and published the first SEOM Consensus on Thromboembolic Disease in Cancer Patients with the aim of increasing awareness of this condition and achieving optimum management of cancer patients. This work programme was completed last year with the publication of the first SEOM Clinical Guidelines on Cancer and Thromboembolic Disease.

In March 2011, in response to this demand and in order to continue with the programme initiated in 2009, the SEOM created a specific Working Group for Cancer and Thrombosis. This Working Group is initially being participated in by over 20 centres across Spain and is made up of various medical specialists, all members of SEOM. This Working Group was started with the aim of raising awareness of thromboembolic disease among medical oncologists and collaborating with other societies and specialists involved in the management of this disease. Three areas have been created within the group, in order to promote three independent working programmes. First of all, a research area to develop a specific clinical and translational research programme. Second, a training education programme addressed at specialist physicians and physicians-in-training. And finally, a care and dissemination programme aimed at creating the proper clinical tools to ensure proper care (clinical guidelines, consensus documents and revisions/updates) and other aspects aimed at improving the level of information for patients and their families on this disease. Naturally, our intention is to collaborate with other national and international working groups involved in this major issue. Therefore we would appreciate it if you could publish this letter in order to make this SEOM initiative widely known. 